\title{
Drosophila Jun kinase regulates expression of decapentaplegic via the ETS-domain protein Aop and the AP-1 transcription factor DJun during dorsal closure
}

\author{
Juan R. Riesgo-Escovar and Ernst Hafen ${ }^{1}$ \\ Zoologisches Institut, Universität Zürich, CH-8057 Zürich, Switzerland
}

\begin{abstract}
During Drosophila embryogenesis, ectodermal cells of the lateral epithelium stretch in a coordinated fashion to internalize the amnioserosa cells and close the embryo dorsally. This process, dorsal closure, requires two signaling pathways: the Drosophila Jun-amino-terminal kinase (DJNK) pathway and the Dpp pathway. We have identified mutations in DJun and show that DJNK controls dorsal closure by activating DJun and inactivating the ETS repressor Aop/Yan by phosphorylation. DJun and Aop regulate dpp expression in the most dorsal row of cells. Secreted Dpp then instructs more ventrally located cells to stretch. Our results provide a causal link between the DINK and Dpp pathways during dorsal closure. Interestingly, in vertebrates, transforming growth factor- $\beta$ and $c$-Jun regulate collagenase gene expression during wound healing, a process that also involves the closing of an epithelial sheath.
\end{abstract}

[Key Words: Jun kinase; TGF- $\beta$; ETS-domain protein; Jun; signaling; development]

Received March 24, 1997; revised version accepted May 7, 1997.

During the development of multicellular organisms, extracellular signals regulate cell division, cell fate, and changes in cell shape. It is important to understand how the large number of signals elicit selectively and reproducibly the correct cellular responses. The availability of genetically tractable organisms in which these processes can be studied in vivo has been instrumental in the elucidation of various signaling pathways (Engelberg et al. 1994; Zipursky and Rubin 1994; Artavanis-Tsakonas et al. 1995; Kayne and Sternberg 1995; Kenyon 1995; Wassarman et al. 1995; Domínguez and Hafen 1996). The results obtained can be broadly applied, because the molecules required in these processes have proven to be conserved between species. The process of dorsal closure during Drosophila embryogenesis is a particularly wellsuited model to study not only individual signaling pathways, but also the interplay between such pathways, because genetic evidence indicates that the closure process is dependent on both Jun kinase and transforming growth factor- $\beta$ (TGF- $\beta$ ) pathways (Affolter et al. 1994; Glise et al. 1995; Riesgo-Escovar et al. 1996; Sluss et al. 1996).

Dorsal closure is defined as the coordinated changes in

${ }^{1}$ Corresponding author.

E-MAIL hafen@zool.unizh.ch; FAX +41 13613185. cell shape in the lateral epithelium that occur at midembryogenesis in Drosophila melanogaster (Campos-Ortega and Hartenstein 1985). Lateral epithelial cells elongate and narrow to cover the dorsal half of the embryo, such that the stretching epithelia meet at the dorsal midline. Previous studies have shown that the onset of dorsal closure can be visualized by actin and myosin accumulation beneath the apical membranes of the most dorsal row of cells of the lateral epithelium, the leading edge cells (Young et al. 1991, 1993). These cells elongate or stretch dorsally over adjacent amnioserosa cells. Subsequently, the ectodermal cells located ventral to the leading edge also stretch until the cells meet at the dorsal midline. Here, the leading edge cells interdigitate to close the ectoderm (Ring and Martinez-Arias 1993; Young et al. 1993).

A large number of embryonic lethal mutations block the process of dorsal closure producing a characteristic dorsal open phenotype. The gene products identified by these mutations can be grouped into two categories of proteins: (1) cytoskeletal and extracellular matrix components (Wieschaus et al. 1984; Wilcox et al. 1989; Young et al. 1993; Fehon et al. 1994; Miyamoto et al. 1995; Borchiellini et al. 1996), and (2) signaling components of the DJNK and Dpp pathways (Jürgens et al. 1984; Nüsslein-Volhard et al. 1984; Affolter et al. 1994; Brummel et al. 1994; Penton et al. 1994; Terracol and 
Lengyel 1994; Glise et al. 1995; Grieder et al. 1995; Harden et al. 1995; Ruberte et al. 1995; Nellen et al. 1996; Riesgo-Escovar et al. 1996; Sluss et al. 1996).

So far, two components of the DJNK pathway have been shown to be required for dorsal closure: Drosophila Jun amino (N)-terminal kinase kinase (DJNKK) encoded by hemipterous (hep) (Glise et al. 1995) and Drosophila Jun amino (N)-terminal kinase (DJNK) encoded by basket (bsk) (Riesgo-Escovar et al. 1996; Sluss et al. 1996). In the absence of DJNKK or DJNK function, lateral epithelial cells fail to stretch. A similar phenotype is observed in embryos with mutations in thick veins $(t k v)$ and punt (put), encoding Decapentaplegic (Dpp) receptors, or mutations in schnurri (shn), encoding a nuclear factor in the Dpp pathway (Jürgens et al. 1984; Nüsslein-Volhard et al. 1984; Affolter et al. 1994; Nellen et al. 1994; Penton et al. 1994; Ruberte et al. 1995). An appraisal of the role of $d p p$ in dorsal closure has been precluded by the fact that $d p p$ plays a vital role earlier in dorsoventral patterning (Irish and Gelbart 1987; Padgett et al. 1987; St. Johnson and Gelbart 1987; Ferguson and Anderson 1992; Jackson and Hoffmann 1994). However, we note that $d p p$ is expressed in the leading edge cells during dorsal closure (St. Johnson and Gelbart 1987).

Here we provide evidence for a functional link between the DJNK and Dpp pathways by showing that the DJNK pathway controls dorsal closure at least in part by regulating $d p p$ expression in the leading edge cells. We show that $d p p$ expression is reduced or absent in leading edge cells of embryos lacking DJNK function. We identify two nuclear targets of the DJNK pathway that are required for dorsal closure, the transcripton factors DJun and the ETS domain repressor Aop/Yan. Mutations in DIun block dorsal closure and reduce $d p p$ expression in the leading edge cells. Conversely, mutations in the ETS domain repressor Aop/Yan lead to $d p p$ overexpression. Expression of an activated Dpp receptor, $\mathrm{Tkv}^{\mathrm{QD}}$, in the lateral epithelium partially restores dorsal closure in the absence of DINK function. These findings suggest that the DJNK pathway governs dorsal closure at least partially by regulating $d p p$ expression via phosphorylation of DJun and Aop.

\section{Results}

Dlun mutation affects dorsal closure

Given the fact that in vertebrate cells, Jun kinase activates the transcription factor Jun (Dérijard et al. 1994; Kallunki et al. 1994), we wondered whether DJNK controls dorsal closure by activation of DJun. Therefore, we searched for embryonic lethal mutations with a dorsal open phenotype in the 46D-E region where DJun has been localized (Perkins et al. 1990; Zhang et al. 1990). 1(2R)IA109 maps to the DJun region and embryos with this mutation are phenotypically very similar to bsk mutant embryos lacking DJNK (Fig. 1C; Nüsslein-Volhard et al. 1984). There is no difference in the strength of the phenotype of embryos homozygous for 1(2R)IA109 or heterozygous over a deficiency that uncovers the locus suggesting that $1(2 R) I A 109$ is a complete loss-of-function mutation. As in bsk mutant embryos, the dorsal open phenotype in this mutant is caused by failure of the lateral epithelial cells to stretch (Fig. 1F). The evidence that $1(2 R) I A 109$ is a complete loss of function allele of D/un is twofold: Rescue experiments involving the repeated induction of a heat inducible D/un transgene (Bohmann et al. 1994) rescued homozygous $1(2 R) I A 109$ embryos to pharate adults. Sequencing of the D/un coding region from the $1(2 R) I A 109$ mutant revealed a single base change at position 651 of the DJun sequence that introduces a stop codon. Therefore, $1(2 R) I A 109$ encodes a truncated DJun protein lacking the bZip DNA-binding and dimerization domain and the amino-terminal phosphorylation sites (Fig. 2). DJun function does not appear to be essential for earlier processes during embryogenesis, because embryos lacking both maternal and zygotic DJun product possess a dorsal open phenotype identical to that of embryos lacking only zygotic DJun function (Fig. 1G).

Genetic evidence that DIun is a critical target in the DINK pathway during dorsal closure

The similarity of the DJNK (Bsk) and the DJun (1/2R)IA109) mutant phenotypes suggests that DJNK controls dorsal closure at least in part by phosphorylation of DJun. This idea is supported by genetic evidence: The partial dorsal open phenotype of weak bsk mutations is strongly enhanced by removing a wild-type copy of D/un or by overexpressing a dominant negative form of DJun [DJun ${ }^{\text {bzIP }}$, (Bohmann et al. 1994)] (Fig. 1H,I). Overexpression of either wild-type DJun or an activated form of vertebrate Jun (Treier et al. 1995) in which the JNK phosphorylation sites have been replaced by acidic residues suppresses the dorsal open phenotype of $b s k$ mutants (Fig. 1J,K). These data indicate that elevated Jun activity can substitute for DJNK function during dorsal closure, supporting the notion that DJNK controls dorsal closure by increasing DJun activity.

D/un is not required for cell fate determination in the developing eye

The similarities of mutant DJun and Bsk embryonic phenotypes indicate that the two genes are required in the same developmental pathway during embryogenesis. However, different requirements for DJun and DJNK during postembryonic development have been reported. Based on mutant eye phenotypes caused by overexpression of dominant negative DJun and activated mammalian c-Jun, it has been proposed that DJun is required for the specification of cell fate in the developing eye (Bohmann et al. 1994; Treier et al. 1995; Peverali et al. 1996). On the other hand, we have shown recently that loss of DJNK function in clones of bsk mutant cells does not interfere with photoreceptor cell specification (RiesgoEscovar et al. 1996). Here we have directly tested the requirement for DJun function in the eye by clonal analysis of the newly identified DIun loss-of-function 


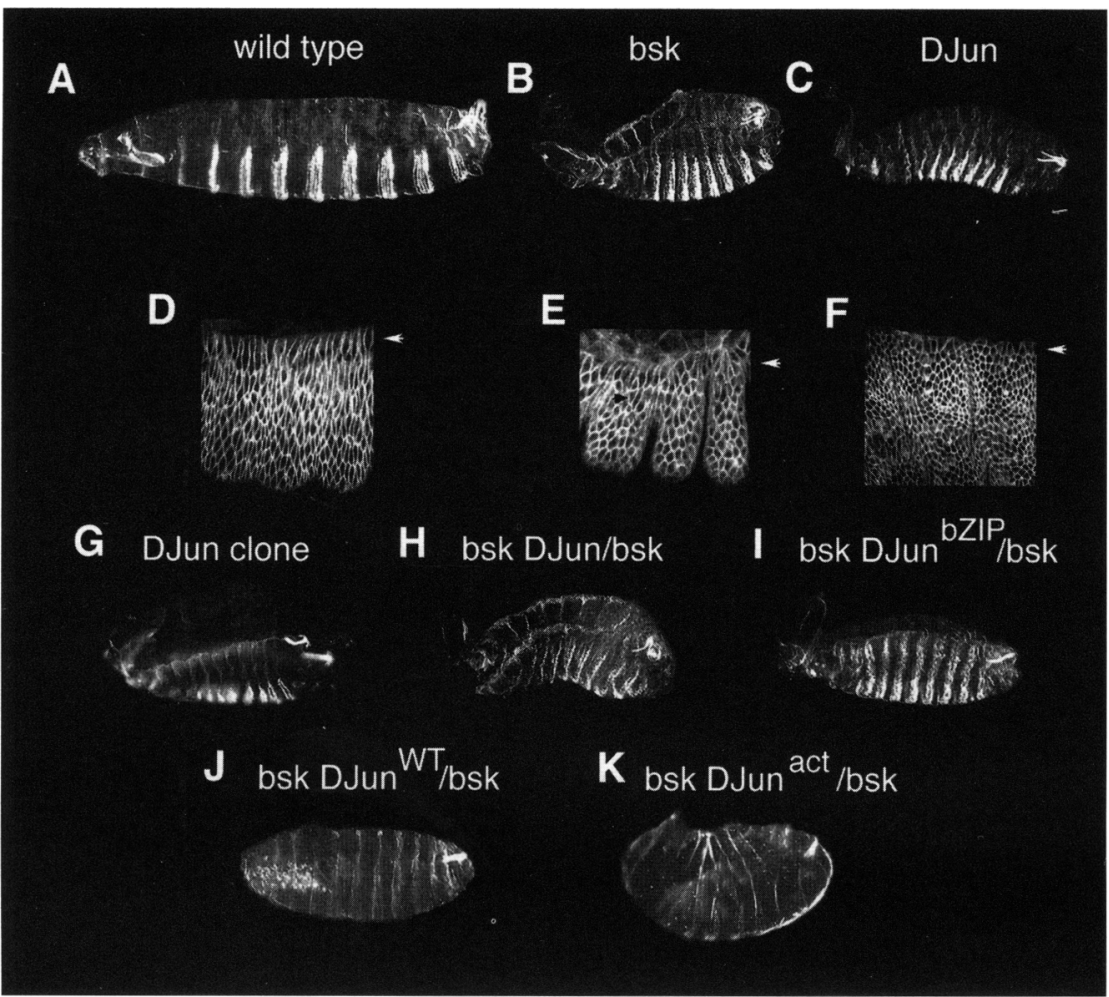

Figure 1. Phenotypic similarities and genetic interactions between 1(2R)IA109/ D/un and bsk mutants. In these and all subsequent Figures, unless noted otherwise, all embryos are shown in a lateral view, with dorsal up and anterior to the left. On the basis of the data presented in the text and in Fig. 2 1(2R)IA109 will be referred to as D/un. Comparison of the embryonic phenotypes of wild-type $(A), b s k^{1}(B)$, and D/un (C) embryos. The cuticle of $b s k^{1}$ embryos is partially closed in the dorsal posterior part $(B)$ whereas that of DIun embryos is completely open dorsally $(C)$. (D-F) A portion of the lateral ectoderm of wild-type, $b s k^{1}$, and D/un mutant embryos, respectively, stained with the anti-Cora antiserum during dorsal closure. Cora protein is not present in the most dorsal membranes of the leading edge cells and this serves as an unambiguous marker to distinguish leading edge cells from adjacent lateral epithelial cells (Fehon et al. 1994). White arrows indicate the position of the leading edge cells. Notice the lack of cell shape changes in the D/un embryo, as opposed to the partial change in cell shape observed in hypomorphic $b s k^{1}$ mutants. The black arrow marks partially stretched lateral cells in the $b s k^{1}$ mutant. $(G)$ An embryo with no maternal or zygotic D/un function. The phenotype is indistinguishable from a D/un zygotic null mutation $(C)$. $(H, I) D / u n$ mutant or expression

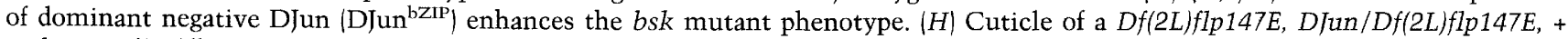
embryo. $D f(2 L) f l p 147 E$ represents a complete loss-of-function mutation for $b s k$. Whereas the dorsal open phenotype of this mutant is somewhat stronger than that shown for $b s k^{1}(B)$, these $D F(2 L) f l p 147 E$ embryos still close on the posterior dorsal side. Removal of one copy of D/un enhances this phenotype such that the entire dorsal side is open. (I) Cuticle of a Df(2L)flp147E, $s E-h s-D / u n^{b Z I P}$ embryo that was heat shocked for one hr at $37^{\circ} \mathrm{C}$ during development. Note that the dorsal aspect of the cuticle is completely open. $(J, K)$ Overexpression of DIun and Jun ${ }^{a c t}$ suppress the bsk phenotype. (J) Cuticle of a Df(2L)flp147E, sE-hs-D/un embryo heat shocked for 1 $\mathrm{hr}$ at $37^{\circ} \mathrm{C}$ during the first $10 \mathrm{hr}$ of embryonic development. The cuticle is completely closed. $(K)$ Cuticle of a $D f(2 L) f l p 147 E$, $s E-h s-J u n^{a c t}$ embryo that has not been heat shocked. The basal expression of Jun ${ }^{\text {act }}$ is sufficient to rescue the dorsal closure phenotype. Note that ectopic expression of D/un and Jun $^{a c t}$ also causes a partial dorsalization of the cuticle as indicated by the severe reduction of the ventral denticle belts.

mutation. Surprisingly, our results indicate that D/un mutant cells contribute to apparently wild-type ommatidial units (Fig. 3). This indicates that DJun, like DJNK, is not required for specification of photoreceptor cell fate in the eye or alternatively, that DJun and DJNK serve a redundant function.

\section{The ETS repressor Aop/Yan is required for dorsal closure}

Amongst the relatively large number of known mutants with defects in dorsal closure we found that aop acts as a strong suppressor of the bsk phenotype (Fig. 4B,D). aop encodes an ETS repressor protein also known as Yan (Lai and Rubin 1992; Tei et al. 1992; Rogge et al. 1995). The phenotype of aop loss of function mutations is pleitropic (Rogge et al. 1995). In the lateral epidermis, the leading edge cells stretch but other cells are irregularly arranged and some are still dividing (Fig. 4E-H). Aop is a target of the MAP kinase encoded by the rolled $(r 7)$ gene in the developing eye and is involved in the specification of photoreceptor cell fate (Brunner et al. 1994; O'Neill et al. 1994). While loss of aop function in the eye results in the differentiation of multiple R7 photoreceptor cells, overexpression of a mutant Aop protein (Aop ${ }^{\text {act }}$ ) which cannot be phosphorylated by MAP kinase, blocks R7 development (Rebay and Rubin 1995). The suppression of the bsk phenotype by aop suggests that the Aop protein also

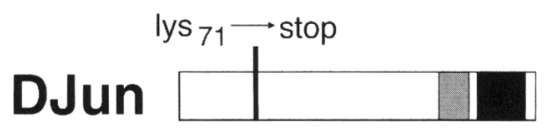

Figure 2. 1(2R)IA109 is a null allele of the D/un locus. A schematic representation of DJun, showing the localization of the mutation in the 1(2R)IA109 allele. (Shaded bar) Basic region; (solid bar) leucine zipper. Sequencing of the mutant DNA showed a single $\mathrm{A}$ to $\mathrm{T}$ substitution at position 651 . The mutant RNA codes for a truncated DJun protein that lacks the DNAbinding and dimerization domain and the three amino-terminal phosphorylation sites thought to be involved in activation of DJun (Peverali et al. 1996). 


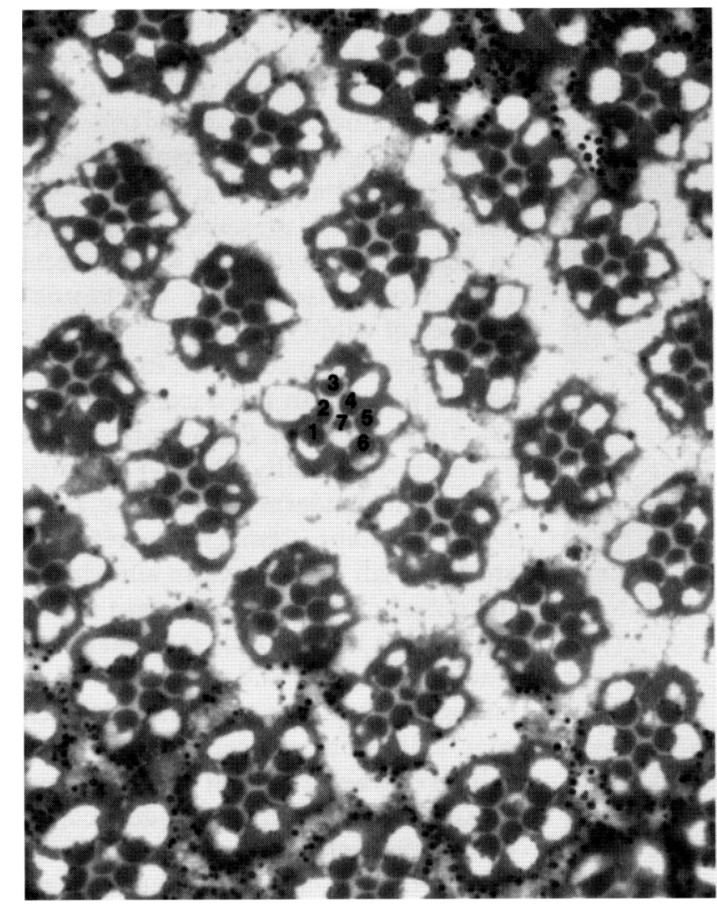

Figure 3. DJun function is not required for the specification of cell fate in the eye. A horizontal section of an eye of a hs-flp; FRT42 DJun/FRT42 $\left.\mathrm{P} / \mathrm{w}^{+}\right]+$fly carrying a large clone of homozygous $D$ Jun mutant cells (marked by the lack of eye pigment) is shown. The compound eye is composed of a hexagonal array of ommatidial units. Each unit consists of eight photoreceptor cells and accessory cells. In this section, seven of the eight photoreceptors are visible. The rhabdomeres (specialized structures of the photoreceptor membranel of the outer photoreceptors (R1-R6) form an asymmetric trapezoid (marked by 1-6 in a mutant, centrally located ommatidium in the figure). The rhabdomere of the R7 photoreceptor occupies the central position in each ommatidium (marked by 7). The specification of photoreceptor cell fate during development is dependent on the activation of the Ras1/Map kinase signaling pathway. The normal number and arrangement of photoreceptors within the D/un mutant clone indicate that there is no absolute requirement for DIun function in the specification of photoreceptor cell fate.

functions as a repressor of dorsal closure. Therefore, we tested whether Aop ${ }^{\text {act }}$ interferes with dorsal closure by expressing it in the lateral epidermis during embryogenesis. Overexpression of Aop ${ }^{\text {act }}$ in the cells of the lateral epithelium prevented dorsal closure and resulted in dead embryos with a strong dorsal open phenotype (Fig. 3J). The genetic interaction and the fact that Aop is phosphorylated by DINK in vitro (Fig. 5) suggest that DINK controls dorsal closure by regulating the activity of the two antagonistic transcription factors: DJun and Aop. Phosphorylation of DJun results in its activation whereas phosphorylation of Aop results in its inactivation.

Expression of dpp in the leading edge cells is regulated by DIun activity

Thus far, we have established that DJun activity and probably also the ETS repressor Aop are regulated by DJNK during dorsal closure. What are the target genes whose expression is regulated by these transcription factors? Two genes, puckered (puc) and $d p p$, are expressed in the leading edge cells during dorsal closure. puc encodes a Cl-100-like MAP kinase phosphatase (A. Gampel, E. Martin-Blanco, and A. Martinez Arias, pers. comm.) and its expression is dependent of DJNK and DJNKK function (Glise et al. 1995; Riesgo-Escovar et al. 1996). Whereas expression of puc at this stage is restricted to the leading edge cells, $d p p$ is expressed in a highly dynamic pattern in other cells as well (St. Johnson and Gelbart 1987; Schwyter et al. 1995). To test whether $d p p$ expression is also controlled by DJNK activity, we examined its expression in embryos with reduced maternal and zygotic bsk function. From 48 embryos undergoing dorsal closure that were derived from females with homozygous bsk mutant germ cells and males heterozygous for a deficiency of $b s k, 26$ embryos had no, or a strongly reduced, expression of $d p p$ in the leading edge cells. The rest of the $d p p$ expression pattern was not affected and, therefore, served as an internal control (Fig. $6 \mathrm{C}, \mathrm{D})$. The frequency of embryos with reduced or absent $d p p$ expression in the leading edge cells correlates well with the fraction of embryos $(50 \%)$ that received the $b s k$ deficiency from the male and, hence, display a bsk mutant phenotype. This indicates that the late expression of $d p p$ in the leading edge cells depends on the activation of the JNK signaling pathway.

As anticipated, $d p p$ expression in the leading edge cells is also dependent on D/un and $a o p$ function: $d p p$ expression is reduced, or absent, in DIun (Fig. 6G) and in embryos expressing aop ${ }^{a c t}$ (Fig. 6H,I). Conversely, $d p p$ expression is expanded in aop mutant embryos (Fig. 6J) and in embryos overexpressing either the activated mammalian c-Jun or wild-type DJun (Fig. 6K,L) . The DJun-induced expansion of $d p p$ expression is suppressed by a deficiency of $b s k$ (Fig. 6M) suggesting that the ectopic DJun function is also dependent on DJNK activity. Whereas expression of DJun ${ }^{\text {bzIP }}$ in wild-type embryos does not reduce $d p p$ expression in the leading edge cells significantly, it blocks $d p p$ expression in embryos lacking zygotic DJNK function (Fig. 6N,O). We note that loss of DJun function affects $d p p$ expression only in the leading edge cells but not, for example, in the visceral mesoderm. In contrast, ubiquitous expression of Jun ${ }^{\text {act }}$ results in an expansion of $d p p$ expression not only in the lateral epidermis, but also in the visceral mesoderm, supporting the idea that results obtained from gain-of-function mutations such as hs-Jun ${ }^{\text {act }}$ may not reflect the normal function of the corresponding gene.

\section{The Dpp signaling pathway is required for dorsal closure}

Given the expression of $d p p$ in the leading edge cells and the dependence of this expression on the activity of the DJNK pathway, we wondered whether Dpp signaling is required for the process of dorsal closure. Owing to the early requirement of $d p p$ function in specification of dor- 


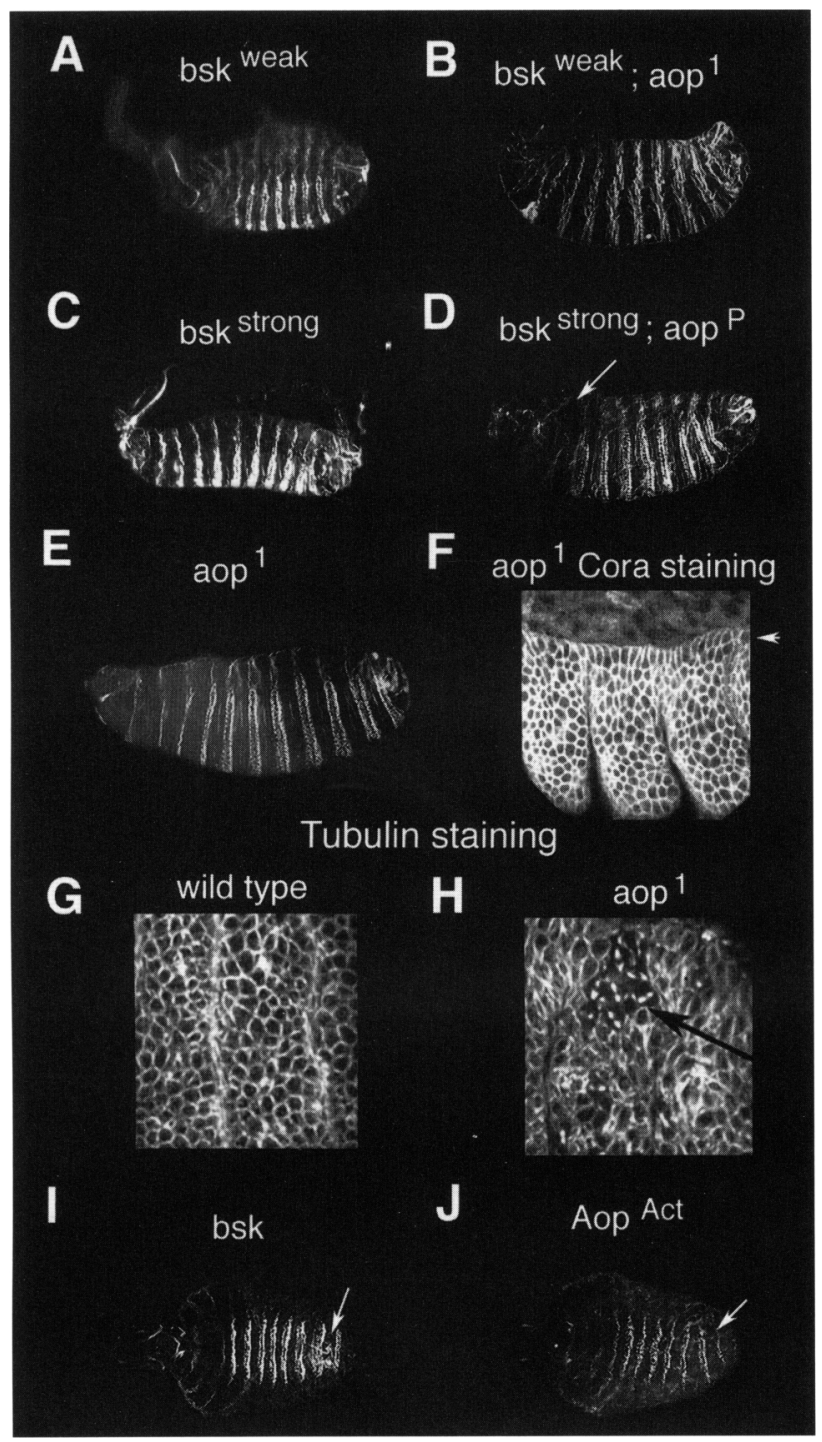

sal-ventral cell fates and the lack of specific mutations that block only the late $d p p$ expression, the analysis of loss-of-function mutations in $d p p$ does not indicate whether $d p p$ function is also required for the late process of dorsal closure. Dorsal open phenotypes have been reported, however, for loss-of-function mutations in the genes coding for two Dpp receptors, tkv and put (Fig. 7A; Affolter et al. 1994; Brummel et al. 1994; Nellen et al. 1994; Penton et al. 1994). Embryos mutant for $t k v$ or put display a milder, but nevertheless similar, phenotype to that observed in bsk mutant embryos (Fig. 7A,C). The mutant embryos exhibit a dorsal hole in the central portion of the embryo. In contrast to bsk mutants, the dorsal anterior and posterior ends are closed normally and appear to be bent inwards (Fig. 7A; Affolter et al. 1994; Brummel et al. 1994; Nellen et al. 1994; Penton et al. 1994).

To examine the cell shape changes during dorsal closure in $t \mathrm{kv}$ and put mutant embryos, the mutant em-
Figure 4. The ETS domain repressor Aop is required for dorsal closure. $(A-D)$ aop acts as a dominant suppressor of bsk. $(A)$ $D f(2 L) f l p 147 E$ mutant embryo. (B) aop ${ }^{1}, D f(2 L) f 1 p 147 E /+$, $D f(2 L) f l p 147 E$ mutant embryo. This embryo has only a small dorsal anterior hole. (C) $b s k^{1} / D f(2 L) f l p 147 E$ embryo derived from a $b s k^{1}$ female germ line clone. Removal of maternal and zygotic bsk function results in a complete dorsal-open phenotype. (D) $a o p^{P}, b s k^{1} /+, D f(2 L) f l p 147 E$ mutant embryo derived from an $a o p^{p}, b s k^{1}$ female germ line. Note that the reduction of aop function during oogenesis results in a marked suppression of the dorsal-open phenotype leaving only a small anterior dorsal hole (white arrow). $(E-H)$ aop mutations show pleiotropic effects in the lateral epithelium during dorsal closure. $(E) \mathrm{Cu}$ ticle of a $a o p^{1}$ homozygous mutant embryo. The dorsal cuticle lacks dorsal hairs and has a convoluted appearance. $(F \mid$ AntiCora stainings of the lateral epithelium of $a o p^{1}$ embryos during dorsal closure. Cells stretch, although they appear more disorganized and are variable in size. The white arrow marks the leading edge cells. $(G, H)$ wild-type $(G)$ and $a o p^{1}(H)$ stage 13 embryos stained with anti-Tubulin. Note the ectopic foci of dividing cells (black arrow) in the $a o p^{1}$ mutant epithelium. $(I, J)$ Expression of aop act phenocopies the bsk mutant phenotype: $(I)$ Ventral view of the cuticle of a $D f(2 L) f l p 147 E$ homozygous embryo; the arrow marks the Filzkörper. (I) Ventral view of a UASAop $^{\text {act }} / 69 \mathrm{~B}$ Gal4 embryo. Except for the rudimentary mouth hooks and Filzkörper (arrow), the cuticular phenotype closely resembles that of bsk mutants shown in $I$.

bryos were stained with the anti-Coracle antiserum (Fehon et al. 1994). Whereas the leading edge cells of $t k v$ and put mutant embryos are extremely elongated and stretched, all other cells of the lateral epidermis are round like the cells in bsk mutant embryos (Fig. 7B). Therefore, in the absence of either of these two receptors for Dpp, the leading edge cells are still able to undergo a change in cell shape. Adjacent, more ventral epithelial cells, however, fail to stretch. Because the expression of $d p p$ in the leading edge cells is controlled by the activity of the DJNK pathway and block of the reception of the Dpp signal in $t k v$ and put mutants results in a failure of the ventral cells to change shape, we suggest that Dpp is involved directly or indirectly in the induction of the cell shape changes in lateral epidermal cells ventral to the leading edge.

To test whether activation of the Dpp signaling pathway is sufficient to induce dorsal closure in the absence of DJNK signaling, we induced ubiquitous ectodermal expression of transgenes encoding $d p p$ or $t k v^{\mathrm{QD}}$ (Nellen et al. 1996), an activated form of the Dpp receptor Tkv. Ectodermal expression of either $d p p$ and $t k v^{Q D}$ in a wildtype background results in dorsalized embryos that undergo normal dorsal closure (Fig. 7D,G). Similarly, the cuticle of bsk embryos was partially dorsalized by the expression of either $d p p$ or $t k v^{Q D}$, but the dorsal hole characteristic of bsk mutant embryos was much reduced in size (Fig. 7E,H,I). Cora staining of bsk mutant embryos ectopically expressing $d p p$ in the ectoderm suggest that lateral ectodermal cells underneath the leading edge cells also stretch (data not shown). Hence, expression of $d p p$ or $t k v^{Q D}$ in $b s k$ mutant embryos resulted in a partial rescue of the dorsal open phenotype. Thus, activation of 


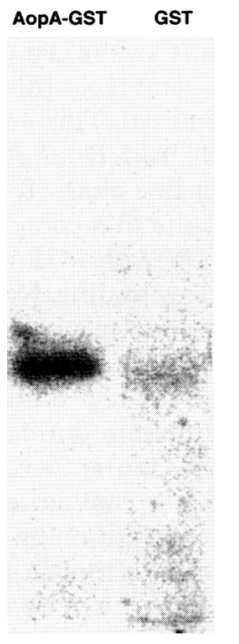

Figure 5. JNK phosphorylates Aop. In vitro kinase assay of JNK with AopA as substrate (Rebay and Rubin 1995). (Lane 1) AopAGST; (lane 2) GST alone. INK was immunoprecipitated from larval cell-free extracts and used for the kinase reactions followed by SDS page and autoradiography. Control experiments in which Mapk and JNK were immunoprecipitated from aliquots of the same larval extracts and used for in vitro kinase assays of bacterially produced AopA showed that both were able to phosphorylate the AopA-GST fusion protein /data not shown). the Dpp signaling pathway can suppress, at least partially, the bsk mutant phenotype. This supports the notion that Dpp secreted by the leading edge cells triggers directly or indirectly the cell shape changes in neighboring cells of the lateral epithelium.

\section{Discussion}

During embryogenesis, the lateral ectoderm closes on the dorsal side by the coordinate lateral elongation of initially round, columnar epithelial cells. It has been shown previously that this process is dependent on the activity of the Drosophila homologs of the stress activated kinases, DJNKK and DINK, encoded by the genes hep and bsk, respectively (Glise et al. 1995; Riesgo-Escovar et al. 1996; Sluss et al. 1996). Here we show that DJNK controls this process by regulating the activity of two transcription factors, DJun and the ETS domain repressor Aop. We show that loss-of-function mutations in DJun block the process of dorsal closure. We further demonstrate that the antagonistic activity of DJun and Aop regulate the localized expression of $d p p$ in the leading edge cells. The reception of the Dpp signal by its receptors Tkv and Put in ectodermal cells ventral to the leading edge cells is essential for these cells to change shape (Fig. 8). These results indicate a causal link between the DINK pathway and the Dpp signaling pathways in the process of dorsal closure.
Figure 6. $d p p$ expression in the leading edge cells is regulated by $b s k, a o p$, and D/un. All panels show embryo whole mounts that had been in situ hy. bridized. For all panels except $E$ the probe was an antisense $d p p$ probe; for $E$ it was an antisense $t k v$ probe that is shown for comparison. $(A, F) d p p$ phase III expression in wild-type embryos (Oregon $\mathrm{R})$ at stage 11 and 13 , respectively. $d p p$ expression in the leading edge cells (arrow in $F$ ) is maintained from germ band extension to the end of dorsal closure. $(B-D) d p p$ expression in bsk mutant backgrounds. (C) $b s k^{1} /+$ embryo derived from a $b s k^{1}$ germ line. This embryo is phenotypically wild-type and shows normal dpp expression. $(C, D) b s k^{1} /$ $D f(2 L) f l p 147 E$ embryo derived from a $b s k^{1}$ germ line. These embryos would develop a strong dorsalopen phenotype and show reduced $(C)$ or absent (arrow in $D$ ) $d p p$ expression. (E) A wild-type embryo hybridized with a $t k v$ antisense probe. $(G) \mathrm{A}$ homozygous DIun ${ }^{I A 109}$ embryo. The arrow marks reduced $d p p$ expression in the leading edge cells. $(H, I) U A S$-Aop ${ }^{a c t}$; 69B-Gal4 embryos. Note the reduced $d p p$ expression in the leading edge cells caused by the expression of Aop ${ }^{\text {act }}$. Conversely, in $I$, an $a o p^{I}$ mutant embryo has ectopic $d p p$ expression (arrow) in rows of cells underneath the leading edge cells. $(K, L)$ Ectopic expression of Jun ${ }^{\text {act }}$ or DJun induces ectopic $d p p$ expression. Embryos in $K-O$ were heat shocked for $1 \mathrm{hr}$, left 3-4 hr to recover, and fixed subsequently. $(K) d p p$ phase III expression domains are expanded in a $h s$-Jun ${ }^{a c t}$ embryo. ( $L$ ) Strong $d p p$ expression in $h s$-DIun embryos. $(M) D f(2 L) f l p 147 E$, $h s$ - $D$ /un embryo. dpp expression is reduced (compare with $L$ ) in the presence of strongly reduced JNK activity. (N) hs-D/un ${ }^{b Z I P}$ and $(O) D f(2 L) f l p 147 E /+, h_{s-D / u n}{ }^{b Z I P}$ embryo. Expression of $J_{u n}{ }^{b Z I P}$ results in reduced $d p p$ expression only in the presence of reduced JNK function $(N, O) .(A, B)$ Dorsolateral views; $(C ; D)$ dorsal views; $(E, O)$ are lateral views.
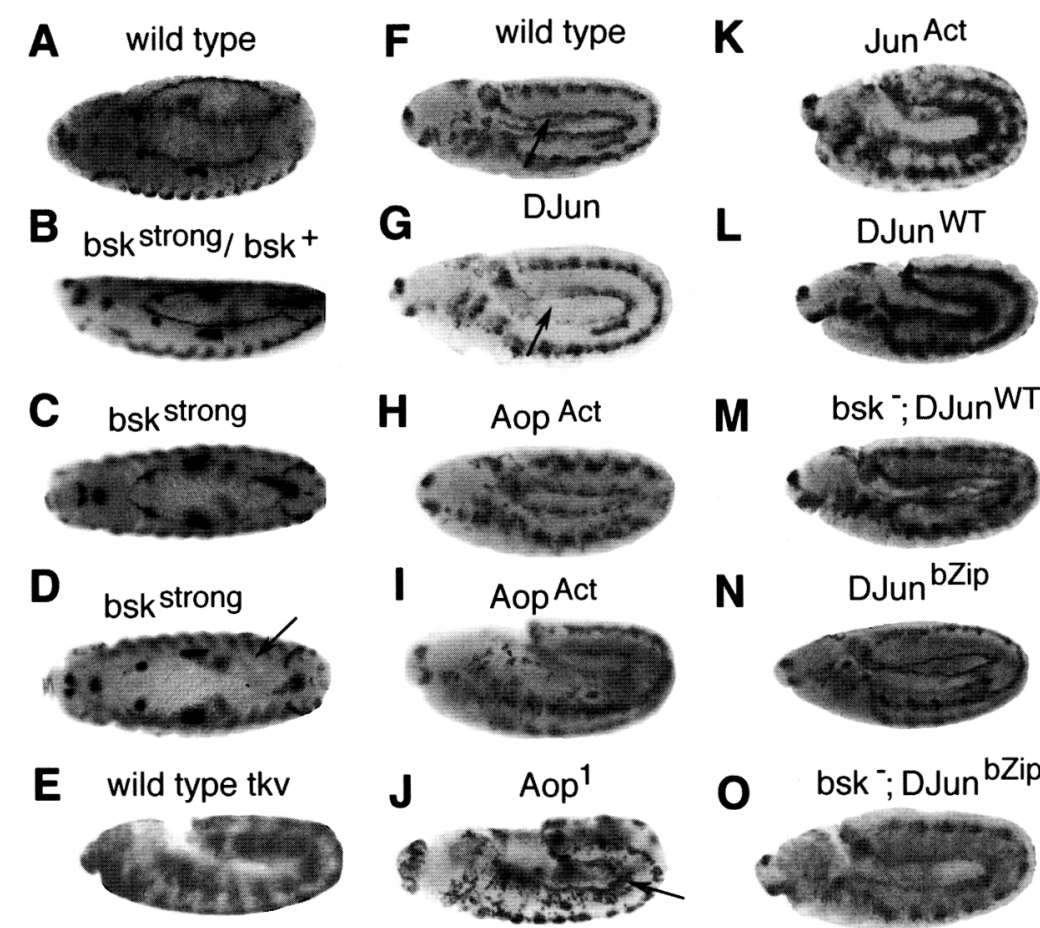

M

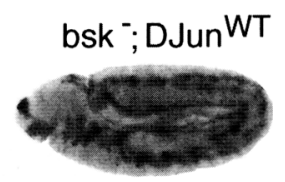

$\mathbf{N}$

DJun bZip
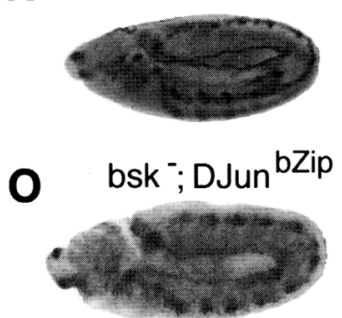


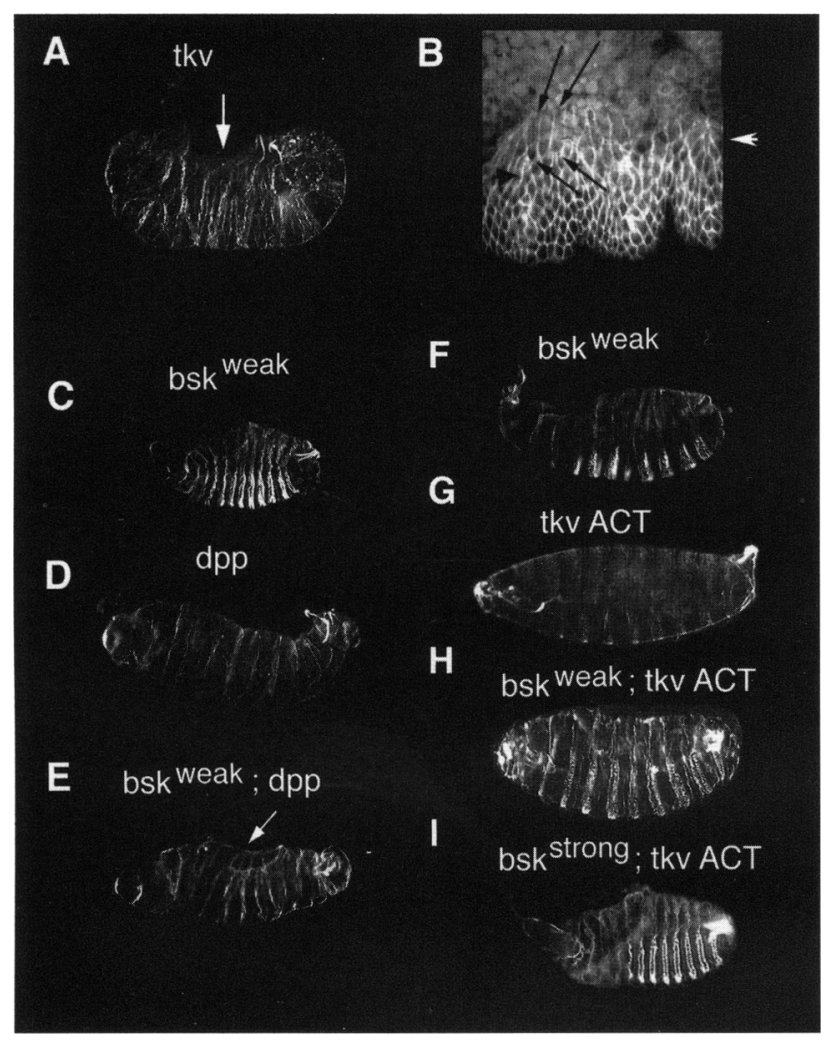

Figure 7. $t k v$ mutants exhibit a partial dorsal closure phenotype. (A) Cuticle of a $t k v^{\text {strII }}$ mutant embryo. Note the centrally located dorsal hole (marked by a white arrow). Unlike bsk and DJun, the most dorsal cuticle is turned inwards and the mouthhooks are retained inside the embryo. The corresponding Cora staining $(B)$ shows that the leading edge cells stretch, but the underlying rows of cells fail to stretch. Two pairs of black arrows mark the dorsal and ventral membranes of two leading edge cells, and the black arrowhead marks cells directly underneath the leading edge cells. $(C-I)$ Expression of $d p p$ or $t k v^{Q D}$ supresses the bsk mutant phenotype. $(C-E)$ and $(G-I)$ sibling embryos from the same experiments, respectively. (C) $D f(2 L) f l p 147 E$ homozygous embryo; (D) UAS-dpp/69B Gal4 embryo; (E) Df(2L)flp147E; UAS-dpp/69B Gal4 embryo. Note the small dorsal hole (arrow) in $(E)$, and compare with $(C) .(F)$ $D f(2 L) f l p 147 E /$ bsk $^{1}$ embryo, (G) UAS-tkv ${ }^{Q D} / 69 B$ Gal4 embryo, and $(H) D f(2 L) f l p 147 E / b s k^{1} ; U A S$ - $t k v^{Q D} / 69 B$ Gal4 embryo. In this weaker $b s k^{-}$mutant combination (compare $C$ with $F$ ) expression of $t k v^{Q D}$ results in almost complete suppression of the bsk mutant phenotype. Both $E$ and $H$ embryos are dorsalized, as are the $D$ and $G$ controls. (I) $b s k^{1} / D f(2 L) f l p 147 E ; U A S-t k v^{Q D} /$ $69 B$ Gal4 embryo derived from a $b s k^{1}$ germ line. Note partial dorsal closure; this phenotype is very similar to bsk zygotic phenotypes.

The similarities between the embryonic phenotypes of DJun and bsk mutants support the idea that the functions of DJun and DJNK are closely linked during embryogenesis. Although it was shown previously that DJNKK and DJNK functions are required for the expression of puc in the leading edge cells (Glise et al. 1995; Riesgo-Escovar et al. 1996), it was not clear whether the DJNK pathway controls dorsal closure via the transcrip- tional regulation of target genes or whether it also acts more directly by phosphorylating components of the cytoskeleton. Because loss of DJun, which we and others have shown to be phosphorylated by DJNK in vitro (Riesgo-Escovar et al. 1996; Sluss et al. 1996), prevents changes in cell shape in much the same way as loss of DINK does, it appears that DJNK controls the cell shape change by the regulation of target genes via DJun.

Although our data suggest that DJun is not essential for cell fate specification in the eye, another transcription factor, the ETS domain repressor Aop, is a target of the MAPK pathway in the eye and of the DINK pathway during dorsal closure in the embryo. In both pathways, Aop acts as a repressor that is inactivated by phosphorylation. The expression of a phosphorylation defective form of Aop prevents photoreceptor cell specification in the eye (Rebay and Rubin 1995), and changes in cell shape in the embryonic lateral epidermis. In the eye, Aop competes with the ETS domain transcriptional activator Pointed P2 (Brunner et al. 1994; O'Neill et al. 1994). Pointed function, however, is not required for dorsal closure because embryos lacking both maternal and zygotic pointed function do not display a dorsal open phenotype and the expression of $d p p$ in the leading edge cells is not altered in these embryos (Mayer \& Nüsslein-Volhard 1988; Riesgo-Escovar \& Hafen, unpubl.). Therefore, although distinct MAP kinase pathways appear to regulate the same repressor in the eye and in the embryo, they activate different activators, Pnt-P2 in the eye and DJun in the embryo. The regulation of a cellular response by the activity of a MAP kinase cascade involving phosphorylation of two antagonistic transcription factors appears to be a common mechanism for reproducible activation of target genes in response to small changes in the activity of the signaling cascades.

The change in cell shape of the lateral epidermis occurs in two phases (Young et al. 1993). In a first phase, the cells of the leading edge begin to stretch dorsally. In a second phase, the remaining cells ventral to the first row change shape. Interestingly, mutants in components of the DINK and the Dpp pathway affect these two phases differently. Whereas loss-of-function mutations in either DJNK or DJun block cell shape changes of all cells, mutations in the genes coding for the Dpp receptors $t k v$ and put block only the second phase. In $t k v$ mutants, the leading edge cells stretch but the remaining cells of the lateral ectoderm fail to change shape. It appears, therefore, that Dpp signaling is required for the induction of cell-shape changes in the ectodermal cells located ventral to the leading edge cells. This is supported by our observation that expression of $d p p$ in the leading edge cells is controlled by the DINK-regulated activities of DJun and Aop.

Is the regulation of $d p p$ expression by DJun and Aop direct? $d p p$ expression during the later stages of embryogenesis, when dorsal closure occurs, has been referred to as phase III expression. An 500-bp enhancer fragment located 400-bp upstream of the transcription start site is necessary and sufficient for $d p p$ ectodermal phase III expression (Schwyter et al. 1995). DJun and Aop may regu- 
Figure 8. Model of the involvement of the DINK and the Dpp pathway in dorsal closure. $(A, B)$ Prior to activation of DJNK by DINKK in the cells of the leading edge $d p p$ expression is repressed by unphosphorylated Aop protein $(A)$. Upon activation of DJNKK by a yet unknown signal possibly transduced by Dcdc42 (Riesgo-Escovar et al. 1996), DJNK is activated (D)NK ${ }^{\text {act}) ~ a n d ~ t r a n s l o c a t e s ~ i n t o ~ t h e ~}$ nucleus, where it phosphorylates Aop and DJun $(B)$. In analogy to phosphorylation by Map kinase (Rebay and Rubin 1995), phosphorylation of Aop by DINK inacitvates the Aop repressor whereas phosphorylation of DJun activates transcription of the target genes $d p p$ and puckered (puc). puc expression in the leading edge cells is regulated by DJNK activity (Riesgo-Escovar et al. 1996). Dpp secreted from the leading edge cells acts as a signal that directly or indirectly induces the ventrally adjacent cells to stretch. $(C)$ In schematic form the changes in cell shape that occur in the lateral epithelium in wild-type embryos, in embryos with mutations in genes coding for components of the DINK pathway or the Dpp pathway. The leading edge cells are hatched. Whereas mutations blocking the DINK pathway including mutations in D/un block stretching of all lateral epithelial cells, Dpp pathway mutants do not affect the stretching of the leading edge cells.

late $d p p$ expression in the leading edge cells by directly binding to sites within this enhancer fragment. Indeed, this enhancer fragment contains multiple consensus sites for binding of AP-1 and ETS proteins. In particular, we found one site where an AP-1- and an ETS-binding site are partially overlapping. Further analysis and dissection of this enhancer will be necessary to establish whether, indeed, DJun and Aop regulate $d p p$ expression by directly binding to this $d p p$ enhancer fragment. There is precedent for AP-1-mediated regulation of TGF $\beta$ transcription: in humans, the TGF- $\beta 1$ promoter is positively controlled by AP-1 (Birchenall-Roberts et al. 1990).

The joint requirement of DJun and Dpp function during embryogenesis appears to be unique to dorsal closure, because other processes involving Dpp such as the establishment of dorso-ventral polarity is not affected in DJun mutants. In vertebrates, the Dpp homolog TGF- $\beta$ and the AP-1 transcription factor act together during wound healing. During this process, TGF- $\beta$ induces collagenase (matrix metalloprotease-1) gene expression in keratinocytes by activating AP-1 (Mauviel et al. 1996). Collagenases are involved in the breakdown of the fibrillary collagen and are important in a number of physiological processes involving connective tissue remodeling such as embryonic development or tumor metastasis (Woessner 1991). It is likely that the breakdown of the extracellular matrix is an important step in the wound healing process which, like dorsal closure, results in the closing of an epithelial sheath. In analogy, DJun activity may control a Drosophila collagenase that dissolves the extracellular matrix, thereby permitting the cells to stretch laterally. A protein immunologically related to the human type-IV collagenase has been identified in Drosophila and shown to accumulate in invasive tumors
(Woodhouse et al. 1994). Furthermore, overexpression of a truncated type IV collagen interferes with dorsal closure (Borchiellini et al. 1996). In D/un mutants, cells may not be able to change shape because they are firmly attached to the basement membrane. Conversely, in $t k v$ mutant embryos the leading edge cells are able to stretch more extremely than in the wild-type. This suggests that in these cells, AP-1 activity is not induced by Dpp but by some other signal, probably from the amnioserosa cells. As in wound healing, Dpp is required to allow more ventrally located cells to change shape, perhaps by inducing AP-1 activity. Although further evidence for the analogy between dorsal closure and wound healing has to await the cloning and characterization of the corresponding collagenase genes, it is an interesting possibility that the connection between the JNK and TGF- $\beta$ signaling pathways has been conserved during evolution. In this respect, it is interesting to note that human TGF- $\beta 1$ transcription is positively regulated by AP-1 activity (Birchenall-Roberts et al. 1990), and that TGF- $\beta 1$ autoinduction is also mediated by AP-1 (Kim et al. 1990).

\section{Materials and methods}

Genetics

put $^{135}$, put $^{P}, \mathrm{UAS}-t k v^{\mathrm{Q} 253 D}\left(=t k v^{Q D}\right), \mathrm{UAS}-d p p, t k v^{\text {strII }}, t k v^{s t r I}$, a CyO and TM3 balancer chromosomes with $\mathrm{y}^{+}$insertions were obtained from R. Burke, D. Nellen and K. Basler (Universität Zürich, Switzerland). sE-hs promoter constructs of wild-type

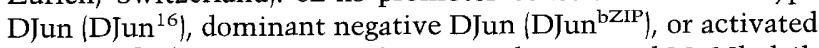

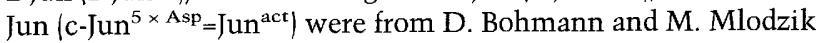
(European Molecular Biology Laboratory, Heidelberg, Germany). UAS-aop act and UAS-aop ${ }^{\mathrm{WT}}$ were from I. Rebay and G. 
Rubin (University of California, Berkeley). $a o p^{1}$ was obtained from E. Brunner (Universität Zürich, Switzerland). $a o p^{P}$ is a new viable $\mathrm{P}$ element allele of $a o p$ isolated in our laboratory (unpubl.). Hypomorphic mutations in aop have been described independently as yan or pokkuri (Lai and Rubin 1992; Tei et al. 1992). Because the first alleles were described by Nüsslein-Volhard et al. (1984) as aop, we have adopted this name in this work. Staining of wild-type embryos with Aop antibody or XGal histochemistry of the hypomorphic $\mathrm{P}$ allele of aop, aop $p^{p}$ show expression of Aop in the lateral epithelium during dorsal closure (data not shown). We obtained an SM6B and TM3 balancer chromosomes with eve-lacZ and $f t z-1 a c Z$ insertions ("blue" balancers) and a deficiency uncovering the DJun locus, $D f(2 R) X 1$, from the Bloomington Stock Center. bsk alleles and I(2R)IA109 were from the Nüsslein-Volhard laboratory. All other alleles and markers are as described (see Lindsley and Zimm 1992; Riesgo-Escovar et al. 1996).

For experiments of $b s k^{-}$homozygotes with UAS- $t k_{V}{ }^{Q D}$ or UAS - $d p p$ under the 69B Gal4 driver (Brand and Perrimon 1993), we prepared stocks that were $y^{-} w^{-}$doubly balanced in the second and third chromosomes over a $b s k^{-}$allele and either a UAS construct or the 69B Gal4 driver in the third chromosome, and crossed flies with the UAS constructs to flies with the Gal4 driver. These experiments were done at $25^{\circ} \mathrm{C}$. We used both a set of second and third chromosome balancers with $1 a c Z$ insertions or with $\mathrm{y}^{+}$insertions, and distinguished the $b s k^{-}$homozygotes UAS-construct/69B driver embryos by lack of $1 \mathrm{acZ}$ staining, or the absence of $y^{+}$. Embryos of all the predicted phenotypes were found at the expected ratios.

For experiments with aop and l(2R)IA109, D/un and mammalian $s E-h s$-Jun constructs, we recombined the mutation or construct on $b_{s k^{-}}$or $1(2 R) I A 109$ chromosomes. In all cases, multiple recombinants were isolated, and more than one recombinant was tested with identical results. To induce DJun or Jun expression, $24 \mathrm{hr}$ egg lays were subjected to heat shocks at $37^{\circ} \mathrm{C}$ for 15,30 , or $60 \mathrm{~min}$. We found that the duration of the heat shock was unimportant (within this range) in all experiments, except for $s E-h s-D / u n^{b Z I P}$, where $1 \mathrm{hr}$ was required to observe the reported phenotypes. In parallel, $b s k^{+}$embryos carrying the same constructs were heat shocked, and the resulting phenotypes were compared. Expression of fun act in a wild-type embryo by a $1 \mathrm{hr}$ heat shock during germ band extension and retraction gives rise to an extreme phenotype akin to dorsalization: Embryos show only naked cuticle and appeared rounded (data not shown). This is consistent with these embryos having strong overexpression of $d p p$ (Fig. 6). $s E-h s-J u n^{a c t}$ embryos without heat shock also show partial dorsalization. Similar, but weaker, phenotypes were observed in heat shocked $h s-D / u n$ embryos. For induction of $D$ Jun mutant clones in the eye and for DIun germ-line clones, $1(2 R) I A 109$ was recombined unto a 42 $F R T$ chromosome (Xu and Rubin 1993). Eye clones were induced by crossing 42 FRT $1(2 R) I A 109$ flies to flies carrying a hs $-f l p$ construct and a $42 F R T, P\left[w^{+}\right]$chromosome. The progeny resulting from this cross was heat shocked to induce flp-mediated recombination. Germ-line clones were induced in females of the genotype hs-flp; 42 FRT l(2R)IA109/42 FRT ovo ${ }^{D}$ by $1 \mathrm{hr}$ heat shocks at late third instar larval stage (Chou and Perrimon 1996). These females were mated to D/un mutant males, or to males carrying a deficiency uncovering DTun. Both crosses gave identical results.

For the UAS-aop ${ }^{a c t} / 69 \mathrm{~B} \mathrm{Gal4}$ experiments, crosses were done at $25^{\circ} \mathrm{C}$ and at $29^{\circ} \mathrm{C}$. In parallel, we examined UAS-aop $p^{W T} / 69 \mathrm{~B}$ driver. The resulting embryos were wild-type. We used both a second and a third chromosome insertions of both types of UASaop constructs. As a control for aop expression and for the $69 \mathrm{~B}$ Gal4 driver, UAS-aop ${ }^{a c t} / 69 \mathrm{~B}$ Gal4 embryos were stained with
Aop antibody. Staining was seen in all ectodermal cells at germ band extension/retraction and dorsal closure stages /data not shown).

\section{Histology}

Embryo X-Gal stainings and antibody stainings were done as described previously in Riesgo-Escovar et al. (1996), except that Cora antibody (Fehon et al. 1994) was used at a 1:2000 dilution. Cora protein expression is normal in $b s k$ (Riesgo-Escovar et al. 1996), $t \mathrm{kv}$, put, and aop mutants (Figs. 1, 3, 7). Aop antibody, a kind gift of I. Rebay and G. Rubin, was used at a 1:10 dilution. Anti $\beta$-Gal antibody from Cappel was used at a 1:2000 dilution. Tubulin stainings were according to Foe (1989). Cuticle preparations were done as reported in Riesgo-Escovar et al. (1996). Digitized images were assembled by use of Adobe Photoshop 3.0 software.

\section{Kinase assays}

Recombinant AopA was purified from bacteria as described (Brunner et al. 1994), and kinase assays were done and quantified as described (Riesgo-Escovar et al. 1996). Recombinant GST was a gift of N. Oellers (Universität Zürich, Switzerland).

\section{In situ hybridization}

Embryos were treated according to Tautz and Pfeifle (1989) with $d p p$ and $t k v$ RNA probes.

\section{Sequencing}

Primers were designed to amplify the D/un coding region between nucleotide 387 and 1350 from genomic DNA of homozygous mutant D/un and control embryos. As a control, the same sequence was amplified from another mutant (pimples) identified in the same mutagenesis screen. The amplified bands were purified and used for sequencing on an ABI Prism 377 sequencer, according to the manufacturer's instructions.

\section{Acknowledgments}

We acknowledge I. Rodriguez for help with in situ hybridization. We especially thank $M$. Domínguez and K. Dücker for discussions and criticisms. We thank I. Rebay and G. Rubin for aop antibody, plasmids and fly stocks, N. Oellers for GST protein, R. Fehon for Cora antibody and S. Leevers, P. Mitchell, K. Basler, and B. Dickson for critical reading of the manuscript. We thank D. Bohmann, D. Nellen, E. Brunner, R. Burke, K. Basler, and M. Mlodzik for fly stocks. Members of the Hafen laboratory provided a very supportive and critical environment. J.R.R.-E. is supported by a Human Frontiers Science Program (HFSP) fellowship, and E.H. by the Swiss National Foundation and the HFSP.

The publication costs of this article were defrayed in part by payment of page charges. This article must therefore be hereby marked "advertisement" in accordance with 18 USC section 1734 solely to indicate this fact.

\section{References}

Affolter, M., D. Nellen, U. Nussbaumer, and K. Basler. 1994. Multiple requirements for the receptor serine/threonine kinase thick veins reveal novel functions of TGF $\beta$ homologs during Drosophila embryogenesis. Development 120: 3105- 
3117.

Artavanis-Tsakonas, S., K. Matsuno, and M.E. Fortini. 1995. Notch signaling. Science 268: 225-232.

Birchenall-Roberts, M.C., F.W. Ruscetti, J. Kasper, H.D. Lee, R. Friedman, A. Geiser, M.D. Sporn, A.B. Roberts, and S.J. Kim. 1990. Transcriptional regulation of the transforming growth factor $\beta-1$ promoter by $\mathrm{v}$-src gene products is mediated through the AP-1 complex. Mol. Cell. Biol. 10: 4978-4983.

Bohmann, D., M.C. Ellis, L.M. Staszewski, and M. Mlodzik. 1994. Drosophila Jun mediates Ras-dependent photoreceptor determination. Cell 78: 973-986.

Borchiellini, C., J. Coulon, and Y. Le Parco. 1996. The function of type IV collagen during Drosophila embryogenesis. Wilhelm Roux's Arch. Dev. Biol. 205: 468-475.

Brand, A.H. and N. Perrimon. 1993. Targeted gene expression as a means of altering cell fates and generating dominant phenotypes. Development 118: 401-415.

Brummel, T.J., V. Twombly, G. Marqués, J.L. Wrana, S.J. Newfeld, L. Attisano, J. Massagué, M.B. O'Connor, and W.M. Gelbart. 1994. Characterization and relationship of Dpp receptors encoded by the saxophone and thick veins genes in Drosophila. Cell 78: 251-261.

Brunner, D., K. Dücker, N. Oellers, E. Hafen, H. Scholz, and C. Klämbt. 1994. The ETS domain protein Pointed-P2 is a target of MAP kinase in the Sevenless signal transduction pathway. Nature 370: 386-389.

Campos-Ortega, J. and V. Hartenstein. 1985. The embryonic development of Drosophila melanogaster. Springer-Verlag, Heidelberg, Germany.

Chou, T.B. and N. Perrimon. 1996. The autosomal FLP-DFS technique for generating germline mosiacs in Drosophila melanogaster. Genetics 144: 1673-1679.

Dérijard, B., M. Hibi, I.H. Wu, T. Barrett, B. Su, T. Deng, M. Karin, and R.J. Davis. 1994. JNK1: A protein kinase stimulated by UV light and $\mathrm{Ha}$-Ras that binds and phosphorylates the c-Jun activation domain. Cell 76: 1025-1037.

Domínguez, M. and E. Hafen. 1996. Genetic dissection of cell fate specification in the developing eye of Drosophila. Semin. Cell \& Dev. Biol. 7: 219-226.

Engelberg, D., C. Klein, H. Martinetto, K. Struhl, and M. Karin. 1994. The UV response involving the Ras signaling pathway and AP-1 transcription factors is conserved between yeast and mammals. Cell 77: 381-390.

Fehon, R.G., I.A. Dawson, and T.S. Artavanis. 1994. A Drosophila homologue of membrane-skeleton protein 4.1 is associated with septate junctions and is encoded by the coracle gene. Development 120: 545-557.

Ferguson, E.L. and K.V. Anderson. 1992. decapentaplegic acts as a morphogen to organize dorsal-ventral pattern in the Drosophila embryo. Cell 71: 451-461.

Glise, B., H. Bourbon, and S. Noselli. 1995. hemipterous encodes a novel Drosophila MAP kinase kinase, required for epithelial cell sheet movement. Cell 83: 451-468.

Grieder, N.C., D. Nellen, R. Burke, K. Basler, and M. Affolter. 1995. schnurri is required for Drosophila dpp signaling and encodes a zinc finger protein similar to the mammalian transcription factor PRDII-BF1. Cell 81: 791-800.

Harden, N., H.Y. Loh, W. Chia, and L. Lim. 1995. A dominant inhibitory version of the small GTP-binding protein Rac disrupts cytoskeletal structures and inhibits developmental cell shape changes in Drosophila. Development 121: 903 914.

Irish, V.F. and W.M. Gelbart. 1987. The decapentaplegic gene is required for dorsal-vental patterning of the Drosophila embryo. Genes \& Dev. 1: 868-879.

Jackson, P.D. and F.M. Hoffmann. 1994. Embryonic expression patterns of the Drosophila decapentaplegic gene: Separate regulatory elements control blastoderm expression and lateral ectodermal expression. Dev. Dynam. 199: 28-44.

Jürgens, G., E. Wieschaus, C. Nüsslein-Volhard, and H. Kluding. 1984. Mutations affecting the pattern of the larval cuticle in Drosophila melanogaster: II. Zygotic loci on the third chromosome. Wilhelm Roux's Arch. Dev. Biol. 193: 183-295.

Kallunki, T., B. Su, I. Tsigelny, H.K. Sluss, B. Dérijard, G. Moore, R. Davis, and M. Karin. 1994. JNK2 contains a specificity-determining region responsible for efficient c-Jun binding and phosphorylation. Genes \& Dev. 8: 2996-3007.

Kayne, P.S. and P.W. Sternberg. 1995. Ras pathways in Caenorhabditis elegans. Curr. Opin. Genet. Dev. 5: 38-43.

Kenyon, C. 1995. A perfect vulva every time: Gradients and signaling cascades in C. elegans. Cell 82: 171-174.

Kim, S.J., P. Angel, R. Lafyatis, K. Hattori, K.Y. Kim, M.B. Sporn, M. Karin, and A.B. Roberts. 1990. Autoinduction of transforming growth factor $\beta 1$ is mediated by the AP- 1 complex. Mol. Cell. Biol. 10: 1492-1497.

Lai, Z.-C. and G. Rubin. 1992. Negative control of photoreceptor development in Drosophila by the product of the yan gene, an ets domain protein. Cell 70: 609-620.

Lindsley, D.L. and G.G. Zimm. 1992. The genome of Drosophila melanogaster. Academic Press, San Diego, CA.

Mauviel, A., K.Y. Chung, A. Agarwal, K. Tamai, and J. Uitto. 1996. Cell-specific induction of distinct oncogenes of the Jun family is responsible for differential regulation of collagenase gene expression by transforming growth factor-beta in fibroblasts and keratinocytes. J. Biol. Chem. 271: 1091710923.

Mayer, U. and C. Nüsslein-Volhard. 1988. A group of genes required for pattern formation in the ventral ectoderm of the Drosophila embryo. Genes \& Dev. 2: 1496-1511.

Miyamoto, H., I. Nihonmatsu, S. Kondo, R. Ueda, S. Togashi, K. Hirata, Y. Ikegami, and D. Yamamoto. 1995. canoe encodes a novel protein containing a GLGF/DHR motif and functions with Notch and scabrous in common developmental pathways in Drosophila. Genes \& Dev. 9: 612-625.

Nellen, D., M. Affolter, and K. Basler. 1994. Receptor serine/ threonine kinases implicated in the control of Drosophila body pattern by decapentaplegic. Cell 78: 225-237.

Nellen, D., R. Burke, G. Struhl, and K. Basler. 1996. Direct and long-range action of a DPP morphogen gradient. Cell 85: 357-368.

Nüsslein-Volhard, C., E. Wieschaus, and H. Kluding. 1984. Mutations affecting the pattern of the larval cuticle in Drosophila melanogaster: I. Zygotic loci on the second chromosome. Wilhelm Roux's Arch. Dev. Biol. 193: 267-282.

O'Neill, E.M., I. Rebay, R. Tjian, and G.M. Rubin. 1994. The activities of two Ets-related transcription factors required for Drosophila eye development are modulated by the Ras/ MAPK pathway. Cell 78: 137-147.

Padgett, R.W., D.R. St. Johnson, and W. Gelbart. 1987. A transcript from a Drosophila pattern gene predicts a protein homologous to the transforming growth factor- $\beta$ family. $\mathrm{Na}$ ture 325: 81-84.

Penton, A., Y. Chen, K. Staehling-Hampton, J.L. Wrana, L. Attisano, J. Szidonya, J.A. Cassill, J. Massagué, and F.M. Hoffmann. 1994. Identification of two bone marrow protein type I receptors in Drosophila and evidence that Brk25D is a decapentaplegic receptor. Cell 78: 239-250.

Perkins, K.K., A. Admon, N. Patel, and R. Tjian. 1990. The Drosophila Fos-related AP-1 protein is a developmentally regulated transcription factor. Genes \& Dev. 4: 822-834.

Peverali, F.A., A. Isaksson, A.G. Papavassiliou, P. Plastina, L.M. Staszewski, M. Mlodzik, and D. Bohmann. 1996. Phosphory- 
lation of Drosophila Jun by the MAP kinase Rolled regulates photoreceptor differentiation. EMBO J. 15: 3943-3950.

Rebay, I. and G.M. Rubin. 1995. Yan functions as a general inhibitor of differentiation and is negatively regulated by activation of the Ras1/MAPK pathway. Cell 81: 857--866.

Riesgo-Escovar, J.R., M. Jenni, A. Fritz, and E. Hafen. 1996. The Drosophila Jun-N-Terminal kinase is required for cell morphogenesis but not for Djun-dependent cell fate specification in the eye. Genes \& Dev. 10: 2759-2768.

Ring, J.M. and A. Martinez-Arias. 1993. Puckered, a gene involved in position-specific cell differentiation in the dorsal epidermis of the Drosophila larva. Development 119: 251259.

Rogge, R., P.J. Green, J. Urano, S. Horn-Saban, M. Mlodzik, B.-Z. Shilo, V. Hartenstein, and U. Baneriee. 1995. The role of yan in mediating the choice between cell division and differentiation. Development 121: 3947-3958.

Ruberte, E., T. Marty, D. Nellen, M. Affolter, and K. Basler. 1995. An absolute requirement for both type II and type I receptors, Punt and Thick Veins, for Dpp signaling in vivo. Cell 80: 889-897.

Schwyter, D.H., J.D. Huang, T. Dubnicoff, and A.J. Courey. 1995. The decapentaplegic core promoter region plays an integral role in the spatial control of transcription. Mol. Cell. Biol. 15: 3960-3968.

Sluss, H.K., Z. Han, T. Barrett, R.J. Davis, and Y.T. Ip. 1996. A INK signal transduction pathway that mediates morphogenesis and an immune response in Drosophila. Genes \& Dev. 10: $2745-2758$.

St. Johnson, R.D. and W.M. Gelbart. 1987. Decapentaplegic transcripts are localized along the dorso-ventral axis of the Drosophila embryo. EMBO I. 6: 2785-2791.

Tautz, D. and C. Pfeifle. 1989. A non-radioactive in situ hybridization method for the localization of specific RNAs in Drosophila embryos reveals translational control of the segmentation gene hunchback. Chromosoma 98: 81-85.

Tei, H., I. Nihonmatsu, T. Yokokura, R. Ueda, Y. Sano, T. Okuda, K. Sato, K. Hirata, S.C. Fujita, and D. Yamamoto. 1992. pokkuri, a Drosophila gene encoding an E-26-specific (Ets) domain protein, prevents overproduction of the R7 photoreceptor. Proc. Natl. Acad. Sci. 89: 6856-6860.

Terracol, R. and J.A. Lengyel. 1994. The thick veins gene of Drosophila is required for dorsoventral polarity of the embryo. Genetics 138: 165-178.

Treier, M., D. Bohmann, and M. Mlodzik. 1995. Jun cooperates with the ETS-domain protein Pointed to induce photoreceptor R7 fate in the Drosophila eye. Cell 83: 753-760.

Wassarman, D.A., M. Therrien, and G.M. Rubin. 1995. The Ras signaling pathway in Drosophila. Curr. Opin. Genet. Dev. 5: 44-50.

Wieschaus, E., C. Nüsslein-Volhard, and G. Jürgens. 1984. Mutations affecting the pattern of the larval cuticle in Drosophila melanogaster: III. Zygotic loci on the X-chromosome and the fouth chromosome. Wilhelm Roux's Arch. Dev. Biol. 193: 296-307.

Wilcox, M., A. DiAntonio, and M. Leptin. 1989. The function of PS integrins in Drosophila wing morphogenesis. Development 107: 891-897.

Woessner, J.J. 1991. Matrix metalloproteinases and their inhibitors in connective tissue remodeling. FASEB T. 5: 2145-2154.

Woodhouse, E., E. Hersperger, W.G. Stetler-Stevenson, L.A. Liotta, and A. Shearn. 1994. Increased type IV collagenase in IgI-induced invasive tumors of Drosophila. Cell Growth Differ. 5: 151-159.

Xu, T. and G.M. Rubin. 1993. Analysis of genetic mosiacs in developing and adult Drosophila tissues. Development 117:
1223-1237.

Young, P.E., T.C. Pesacreta, and D.P. Kiehart. 1991. Dynamic changes in the distribution of cytoplasmic myosin during Drosophila embryogenesis. Development 111: 1-14.

Young, P.E., A.M. Richman, A.S. Ketchum, and D.P. Kiehart. 1993. Morphogenesis in Drosophila requires nonmuscle myosin heavy chain function. Genes \& Dev. 7: 29-41.

Zhang, K., J.R. Chaillet, L.A. Perkins, T.D. Halazonetis, and N. Perrimon. 1990. Drosophila homolog of the mammalian jun oncogene is expressed during embryonic development and activates transcription in mammalian cells. Proc. Natl. Acad. Sci. 87: 6281-6285.

Zipursky, L.S. and G.M. Rubin. 1994. Determination of neuronal cell fate: Lessons from the R7 neuron in Drosophila. Annu. Rev. Neurosci. 17: 373-397 


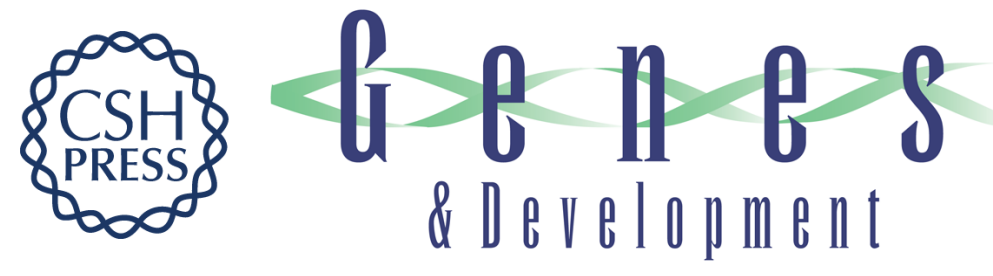

\title{
Drosophila Jun kinase regulates expression of decapentaplegic via the ETS-domain protein Aop and the AP-1 transcription factor DJun during dorsal closure.
}

\author{
J R Riesgo-Escovar and E Hafen
}

Genes Dev. 1997, 11:

Access the most recent version at doi:10.1101/gad.11.13.1717

References This article cites 58 articles, 27 of which can be accessed free at:

http://genesdev.cshlp.org/content/11/13/1717.full.html\#ref-list-1

\section{License}

Email Alerting Service

Receive free email alerts when new articles cite this article - sign up in the box at the top right corner of the article or click here.

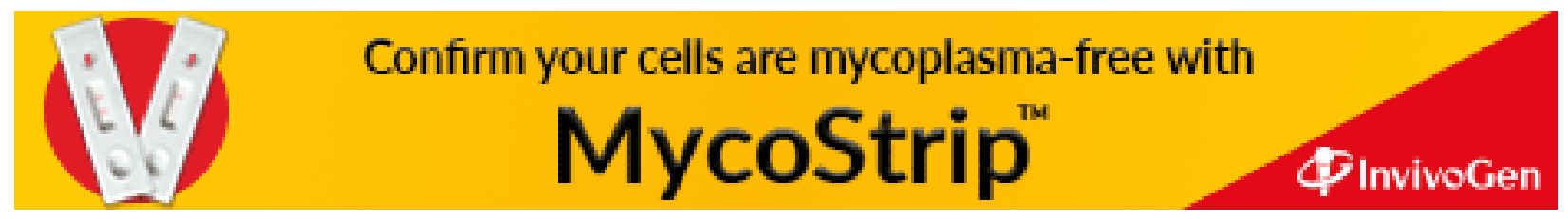

